\title{
Evolução do perfil das internações psiquiátricas pelo Sistema Único de Saúde em Minas Gerais, Brasil, 2001-2013
}

\author{
The evolution of the profile of psychiatric admissions \\ via the Unified Health System in Minas Gerais, Brazil, 2001-2013
}

Ana Paula Martins Lara ${ }^{1}$

Fernando Madalena Volpe ${ }^{2}$

${ }^{1}$ Programa de PósGraduação em Promoção da Saúde e Prevenção da Violência, Faculdade de Medicina, Universidade Federal de Minas Gerais. Av. Prof. Alfredo Balena 190/8, Santa Efigênia. 30130-100 Belo Horizonte MG Brasil. anapaulamlara@gmail.com

${ }^{2}$ Fundação Hospitalar do

Estado de Minas Gerais.

Belo Horizonte MG Brasil.
Abstract The scope of this article is to analyze the evolution of the profile of psychiatric admissions via the Unified Health System in psychiatric hospitals of the State of Minas Gerais, Brazil, between 2001 and 2013. Data were obtained from the Information Technology Department of the Unified Health System. The analyses of trends were conducted by regression procedures, in which the independent variable was the year, and the dependent variables were the patients (sex, age, diagnosis) and admission characteristics (city, hospital administrative status, length of internment). A total of 202,188 admissions to 25 hospitals were appraised. There were significant changes in the diagnostic profiles of psychiatric admissions during the period under scrutiny, notably an increase in the proportion of admissions for substance abuse-related disorders and a reduction for psychotic disorders. This study is in tune with the context of the reform of mental healthcare in Minas Gerais, providing relevant input to support the mental health policies towards universalization, humanization and the overcoming of inequalities in access to health services.

Key words Mental health, Psychiatric hospitals, Length of internment, Deinstitutionalization, Health information systems
Resumo O objetivo deste artigo é analisar a evolução no perfil das internações psiquiátricas pelo Sistema Único de Saúde em hospitais psiquiátricos do Estado de Minas Gerais entre 2001 e 2013. Os dados foram obtidos através do Departamento de Informática do SUS. A análise de tendência deu-se através de procedimentos de regressão, em que o ano foi variável independente e as variáveis dependentes foram as características dos pacientes (sexo, idade, diagnóstico) e das internações (localidade, natureza jurídica do hospital, tempo de permanência). Foram incluídas 202.188 internações em 25 hospitais. Houve alterações significativas no perfil nosológico das internações psiquiátricas, com elevação da proporção das internações por transtornos ligados ao abuso de substâncias e redução por transtornos psicóticos. O estudo se insere no contexto da reforma da assistência à saúde mental em Minas Gerais, produzindo informações relevantes para subsidiar as políticas de saúde mental na direção à universalização, humanização e superação das desigualdades de acesso aos serviços de saúde.

Palavras-chave Saúde mental, Hospitais psiquiátricos, Tempo de internação, Desinstitucionalização, Sistemas de informação em saúde 


\section{Introdução}

Diante das mudanças nos marcos legais e das diretrizes assistenciais ocorridas no Brasil nas últimas duas décadas, o papel do hospital psiquiátrico, entre os diversos dispositivos de atenção à saúde mental disponíveis, se modifica, bem como as trajetórias terapêuticas dos usuários desses serviços. Concretamente, no Brasil, o número de leitos psiquiátricos reduziu-se de 51.393 para 29.958, de 2002 até $2012^{1}$, ao mesmo tempo em que o número de Centros de Atenção Psicossocial (CAPS) saltou de 424, no ano de 2002, para o somatório de $1.803 \mathrm{em} 2012^{1}$.

Além disso, outros dispositivos tiveram muita importância nesse processo de estruturação da rede de atenção à saúde mental e de desinstitucionalização no país, tais como: o Centro de Atenção Psicossocial (CAPS), o Serviço Residencial Terapêutico (SRT), o Serviço Hospitalar de Referência (SHR), o desenvolvimento do Programa Nacional de Avaliação do Sistema Hospitalar/Psiquiatria (PNASH/Psiquiatria), do Programa Anual de Reestruturação da Assistência Hospitalar Psiquiátrica no SUS (PRH) e do Programa De Volta para Casa ${ }^{2}$. Nessa perspectiva, foi também instituída a Rede de Atenção Psicossocial (RAPS), a qual dispõe sobre o cuidado integral centrado nos territórios ${ }^{3}$.

Essas mudanças nas diretrizes assistenciais possivelmente se acompanham de alterações nas demandas e também nas práticas terapêuticas, ambas objetivamente observáveis. Por exemplo, em dois hospitais psiquiátricos públicos de Belo Horizonte, Coelho et al. ${ }^{4}$ descreveram importantes alterações dos perfis das internações durante uma década, em que se observou aumento no número de internações, redução dos tempos de permanência, sem alteração das taxas de reinternação. Outro interessante achado foi a alteração no perfil nosológico prevalente, com a relativa elevação das internações por transtornos ligados ao abuso e dependência de substâncias psicoativas, superando as internações por transtornos psicóticos ${ }^{4}$.

Os escassos estudos brasileiros que avaliam os perfis demográficos e nosológicos das internações psiquiátricas ao longo do tempo ${ }^{5,6}$ demonstram que há heterogeneidade nas mudanças descritas, dependendo do local e do tipo de hospital analisado. Diante disso, não é aconselhável extrapolar os achados de Coelho et al. ${ }^{4}$ para a totalidade do estado de Minas Gerais, que engloba hospitais de diferentes naturezas e organizações de rede de assistência desiguais.
A Organização Mundial de Saúde (OMS) preconiza que a melhoria da assistência à saúde mental implica investimento na produção de dados concretos sobre os serviços e recursos. Nessa conformidade poderão ser definidas estratégias de avaliação contínua das atividades desempenhadas e das mudanças instituídas ${ }^{7,8}$. Eventuais mudanças observadas nas características das internações psiquiátricas conseguintes à implementação das novas políticas de assistência são, assim, relevantes para a reflexão sobre seus resultados.

Nesse contexto, o presente estudo descreve a evolução do perfil das internações pelo SUS nos hospitais psiquiátricos especializados no estado de Minas Gerais, entre os anos 2001 e 2013.

\section{Métodos}

Trata-se de estudo observacional, descritivo, de séries temporais.

\section{Caracterização da amostra}

A definição do início do período da pesquisa foi assim delimitada por ser $2001 \mathrm{o}$ ano de publicação da Lei Federal n ${ }^{\circ}$ 10.216. Esta é considerada um marco histórico e regulatório, pois determinou o redirecionamento da assistência em saúde mental nos anos que se seguiram, priorizando a atenção extra-hospitalar.

O estudo foi realizado a partir de dados secundários disponibilizados pelo DATASUS ${ }^{9}$, a partir do Sistema de Informações Hospitalares do Sistema Único de Saúde (SIH-SUS). Os dados obtidos são referentes às Autorizações para Internação Hospitalar (AIH) faturadas dos hospitais psiquiátricos especializados em Minas Gerais, de internações ocorridas de $1^{\circ}$ de janeiro de 2001 até 31 de dezembro de 2013.

Dos hospitais selecionados, existem aqueles que foram desativados no período entre os anos de 2001 e 2013, porém, para fins do estudo de séries temporais, é fundamental a inclusão das informações sobre as internações nos anos em que estiveram em funcionamento. Essas instituições englobam a totalidade dos leitos em hospitais psiquiátricos disponíveis pelo SUS em Minas Gerais, com exceção do hospital psiquiátrico especializado na infância e adolescência ${ }^{10}$. A Tabela 1 apresenta a listagem dos hospitais psiquiátricos mineiros onde houve internações pelo SUS entre 2001 e 2013. 
Tabela 1. Caracterização dos hospitais psiquiátricos com internações pelo SUS em Minas Gerais, 2001-2013.

\begin{tabular}{|c|c|c|c|c|c|}
\hline Hospital & Município & $\begin{array}{l}\text { Natureza } \\
\text { Jurídica }\end{array}$ & $\begin{array}{l}\text { No leitos } \\
\text { SUS/2002 }\end{array}$ & $\begin{array}{l}\text { No leitos } \\
\text { SUS/2013 }\end{array}$ & Ano de fechamento \\
\hline Clinica Serra Verde & Belo Horizonte & Privada & 303 & 0 & 2012 \\
\hline Clinica Mantiqueira & Barbacena & Privada & 199 & 82 & $\begin{array}{l}\text { Em processo de } \\
\text { desisnstitucionalização }\end{array}$ \\
\hline $\begin{array}{l}\text { Fundação Beneficente São João } \\
\text { da Escócia - Otto Krakauer }\end{array}$ & Passos & Privada & 138 & 120 & Em funcionamento \\
\hline $\begin{array}{l}\text { Clinica Neuropsiquiátrica de } \\
\text { Alfenas }\end{array}$ & Alfenas & Privada & 159 & 0 & 2009 \\
\hline $\begin{array}{l}\text { Organização Hospitalar } \\
\text { Psiquiátrica - Prontomente }\end{array}$ & Montes Claros & Privada & 120 & 0 & 2013 \\
\hline $\begin{array}{l}\text { Sanatório Espirita José Dias } \\
\text { Machado }\end{array}$ & Ituiutaba & Privada & 51 & 51 & Em funcionamento \\
\hline Casa de Saúde Santa Izabel & Barbacena & Privada & 110 & 76 & Em funcionamento \\
\hline Casa de Saúde Dr. Aragão Vilar & Juiz de Fora & Privada & 170 & 160 & 2014 \\
\hline Casa de Saúde Esperança & Juiz de Fora & Privada & 170 & 180 & 2014 \\
\hline Hospital São Marcos & Juiz de Fora & Privada & 120 & 101 & 2013 \\
\hline Hospital São Domingos & Juiz de Fora & Privada & 120 & 128 & 2013 \\
\hline $\begin{array}{l}\text { Clínica Psiquiátrica Pinho } \\
\text { Masini }\end{array}$ & Juiz de Fora & Privada & 90 & 0 & 2011 \\
\hline Casa de Saúde Paulo Menicucci & Lavras & Privada & 164 & 0 & 2013 \\
\hline Clinica São José & Leopoldina & Privada & 268 & 150 & Em funcionamento \\
\hline $\begin{array}{l}\text { Fundação Sanatório Gedor } \\
\text { Silveira }\end{array}$ & $\begin{array}{l}\text { São Sebastião } \\
\text { do Paraiso }\end{array}$ & Privada & 170 & 160 & Em funcionamento \\
\hline Centro Espirita Uberabense & Uberaba & Privada & 158 & 120 & Em funcionamento \\
\hline $\begin{array}{l}\text { Congregação das Irmãs } \\
\text { Hospitaleiras do Sagrado } \\
\text { Coração de Jesus- Bento Menni }\end{array}$ & Divinópolis & Privada & 90 & 87 & Em funcionamento \\
\hline Hospital Galba Veloso & Belo Horizonte & Pública & 153 & 145 & Em funcionamento \\
\hline Instituto Raul Soares & Belo Horizonte & Pública & 120 & 108 & Em funcionamento \\
\hline $\begin{array}{l}\text { Centro Hospitalar Psiquiátrico } \\
\text { de Barbacena }\end{array}$ & Barbacena & Pública & 456 & 240 & Em funcionamento \\
\hline Instituto Psicominas & Belo Horizonte & Privada & 150 & 0 & 2003 \\
\hline Clinica Pinel & Belo Horizonte & Privada & 220 & 0 & 2003 \\
\hline $\begin{array}{l}\text { Clinica Nossa Senhora de } \\
\text { Lourdes }\end{array}$ & Belo Horizonte & Privada & 280 & 0 & 2008 \\
\hline Sanatório Barbacena & Barbacena & Privada & 103 & 0 & 2003 \\
\hline Casa de Saúde Xavier & Barbacena & Privada & 110 & 0 & 2004 \\
\hline
\end{tabular}

Fonte: Coordenação Geral de Saúde Mental Álcool e outras Drogas (CGMAD) e Cadastro Nacional de Estabelecimentos de Saúde (CNES/DATASUS -Agosto, 2015) - Organizado pelos autores.

\section{Adequação do banco de dados de AIH}

O sistema de registro das AIHs psiquiátricas no DATASUS apresenta uma peculiaridade: internações prolongadas são registradas com múltiplas AIHs.

Apesar da orientação do Ministério da Saúde para que AIHs subsequentes na mesma internação recebam numeração igual, muitas vezes a numeração registrada é diferente, o que dificulta a integração das informações. Assim, uma mesma internação pode possuir diversas $\mathrm{AlHs}^{11}$. Além disso, os arquivos disponibilizados publicamente pelo DATASUS não contêm dados de identificação do paciente (nome, identidade, CPF). Portanto, para integrar as AIHs referentes a uma mesma internação foram necessárias técnicas de processamento de dados por similaridade.

A técnica utilizada para esse agrupamento envolveu a unificação dos arquivos de dados mensais em um único banco de dados. Os registros foram então classificados por hospital, em segui- 
da por data de nascimento e, finalmente, por data de internação. A partir de expressões programadas no Excel, foram identificadas as AIHs seriadas de uma mesma internação quando as seguintes condições foram atendidas: a) mesmo hospital; b) mesma data de nascimento; c) mesmo sexo; d) data de internação da AIH subsequente igual à data de saída da AIH anterior ou data de internação da AIH subsequente igual à data de saída da AIH anterior mais um dia ou data de internação da AIH subsequente englobada no período de internação da AIH anterior. Com esse procedimento de agrupamento, do total inicial de 484.376 AIHs, chegou-se a 202.188 internações registradas no período do estudo.

Uma vez identificadas as AIHs seriadas de uma mesma internação, estas foram agrupadas consolidando-se as informações variáveis da seguinte forma: a) a data de internação considerada foi a menor data da série; b) a data de saída considerada foi a maior data da série; c) o tempo de permanência foi calculado pela subtração entre a data de saída e a data da internação após a consolidação; d) a idade considerada foi a do primeiro registro da série; e) o diagnóstico (segundo a CID-10) $)^{12}$ considerado foi o do último registro da série. Procedimento semelhante foi descrito por outros autores que encontraram a mesma dificuldade ao utilizar o banco de dados do SIH-SUS para análise das internações psiquiátricas $^{5,13}$.

\section{Variáveis do estudo}

Neste estudo, foram utilizadas as seguintes variáveis: a) sexo; b) idade (em anos); c) diagnóstico principal (por grupo da CID-10); d) data de internação; e) data de saída; f) tempo de permanência (em dias, resultantes da subtração da data de saída e a data de internação); g) nome do hospital; h) natureza jurídica do hospital (público ou privado).

\section{Análise dos dados}

Primeiramente, procedeu-se à descrição da amostra total, segundo as variáveis demográficas e clínicas de interesse.

Para as análises de séries temporais, as tendências foram estudadas a partir de regressões lineares ou de outros modelos estatísticos (quadrático ou cúbico), de acordo com as distribuições apresentadas, tendo como variável-resposta o número ou a proporção anual de internações e como variável independente o ano, com nível de confiança de $95 \%$. Foram efetuadas análises de séries temporais por sexo, por idade, por grupo diagnóstico e por tempo de permanência. Análises da distribuição dos resíduos foram conduzidas para verificar a adequação dos modelos escolhidos.

A análise da evolução do tempo de permanência ao longo do período do estudo se deu após a exclusão das internações com duração superior a 365 dias, bem como as ocorridas no último ano da série. Esse procedimento visou a equalizar os riscos de permanecer internado, independentemente da data de admissão. Essa variável apresentou distribuição não gaussiana, portanto, foram utilizados os seus valores medianos para fins de comparação.

Os subgrupos de hospitais públicos e privados foram analisados separadamente quanto às evoluções temporais do tempo de permanência e quanto às mudanças nos perfis nosológicos.

\section{Considerações éticas}

O presente estudo é parte integrante de um projeto maior intitulado: Projeto Epiquali - diagnóstico epidemiológico e administrativo da rede hospitalar psiquiátrica que presta assistência a usuários do SUS em Minas Gerais. O Projeto Epiquali foi aprovado pelo Comitê de Ética e Pesquisa (COEP) da Universidade Federal de Minas Gerais (UFMG) em 23 de janeiro de 2014.

\section{Resultados}

A amostra foi composta de 202.188 internações ocorridas no período de 2001 a 2013. As características dessas admissões estão apresentadas na Tabela 2.

No início do período observacional, 2001, Minas Gerais contava com três hospitais públicos e 22 hospitais privados conveniados, prestando assistência hospitalar pelo SUS. Ao longo dos 13 anos do estudo, saíram da série 10 hospitais privados, por fechamento ou descredenciamento.

O número total de internações apresentou significativa redução no período, seguindo uma tendência cúbica em que as reduções mais aceleradas se deram nos primeiros e nos últimos três anos de observação (Figura 1).

Excluindo as internações de longa permanência (> 365 dias), o tempo mediano de permanência hospitalar oscilou suavemente entre $28 \mathrm{e}$ 31 dias ao longo do período de estudo. Contudo, 
Tabela 2. Características das internações nos hospitais psiquiátricos de Minas Gerais, 2001-2013. (N=202.188).

\begin{tabular}{|c|c|c|}
\hline & $\mathbf{N}$ & $\%$ \\
\hline Sexo Masculino & 129.711 & 64,2 \\
\hline Idade (média \pm DP) & 39,1 & $( \pm 12,5)$ \\
\hline \multicolumn{3}{|l|}{ Grupo Diagnóstico } \\
\hline Tr mentais orgânicos, incluindo sintomáticos - F00-F09 & 16.574 & 8,2 \\
\hline $\operatorname{Tr}$ decorrentes do abuso/dependência de substância psicoativa - F10-F19 & 57.702 & 28,5 \\
\hline Esquizofrenia, tr esquizotípicos e delirantes - F20-F29 & 88.155 & 43,6 \\
\hline Tr do humor (afetivos) - F30-F39 & 30.094 & 14,9 \\
\hline Tr neuróticos, relacionados ao estresse e somatoformes - F40-F49 & 2.547 & 1,3 \\
\hline $\begin{array}{l}\text { Síndromes comportamentais associadas a perturbações fisiológicas e fatores de } \\
\text { risco - F50-F59 }\end{array}$ & 218 & 0,1 \\
\hline $\mathrm{Tr}$ de personalidade e de comportamentos em adultos - F60-F69 & 1.533 & 0,8 \\
\hline Retardo mental - F70-F79 & 5.156 & 2,6 \\
\hline Outros & 209 & 0,1 \\
\hline Tempo de permanência mediano [min;max] & 30 & {$[0 ; 4.747]$} \\
\hline
\end{tabular}

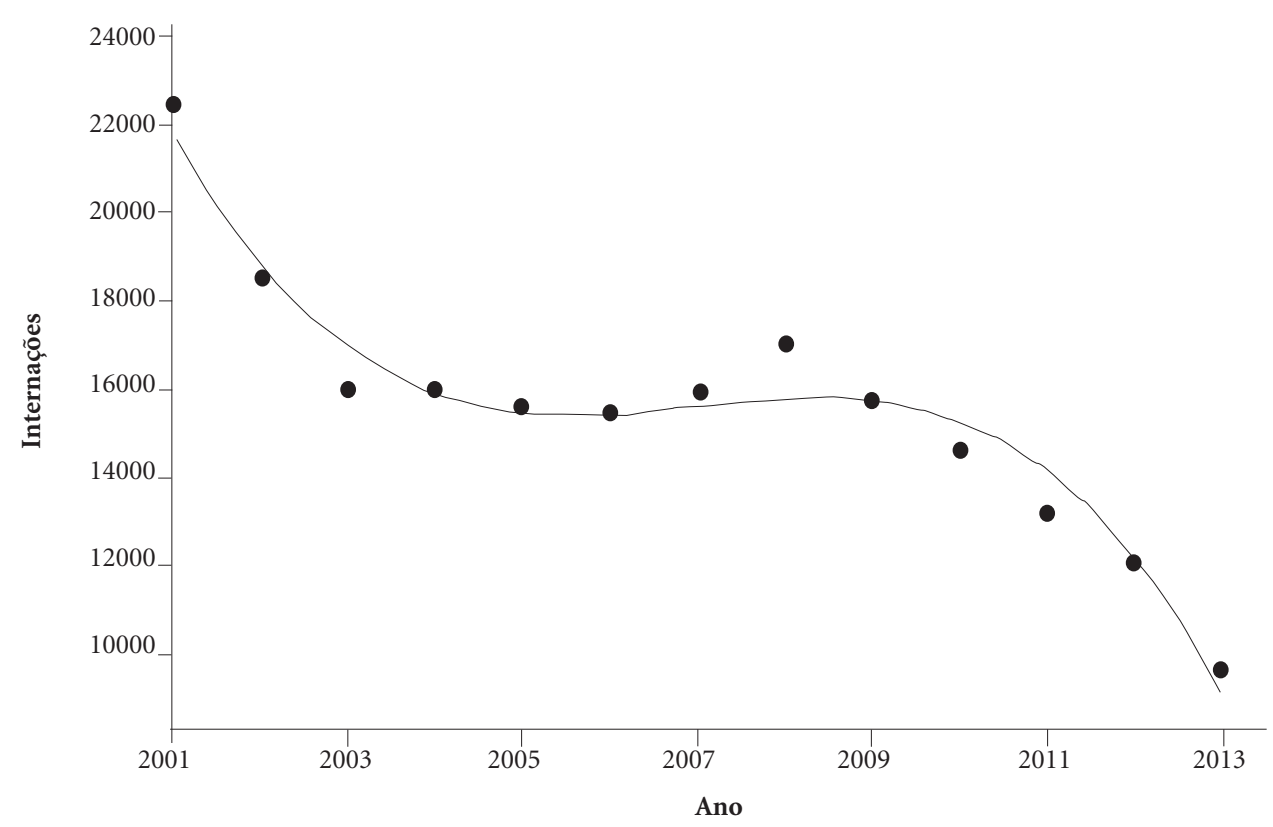

Tendência cúbica, $\mathrm{R}^{2}=94,5 \% ; \mathrm{P}<0,001$.

Figura 1. Evolução do número de internações em hospitais psiquiátricos de Minas Gerais, 2001-2013. $(\mathrm{N}=202.188)$.

observou-se evolução diferenciada nos hospitais públicos e privados entre 2001 e 2012, ocorrendo clara redução nos primeiros e elevação nos segundos (Figura 2).

As internações prolongadas, acima de 365 dias, representaram 5,3\% $(\mathrm{n}=1.292)$ dos casos em 2001 e reduziram-se para $1,3 \%(n=151)$ dos casos em 2012.

No período de 2001 a 2013, a idade média dos pacientes internados se manteve constante $(\mathrm{F}=$ $2,13 ; \mathrm{p}=0,145)$. A proporção de internações do sexo masculino apresentou ligeira, mas significa- 
tiva tendência à elevação, de 63,3 a 64,5\% (F = $7,8 ; \mathrm{p}=0,018)$ no período.

Houve alterações significativas no perfil nosológico das internações psiquiátricas no intervalo do estudo. As proporções de internações por transtornos mentais orgânicos (grupo da CID-10 F0) apresentaram suave elevação até a metade do período, passando de 8,8 a 9,2\%, seguindo-se uma tendência à redução de até $6,2 \%$. As internações por transtornos ligados ao uso de substâncias (F1) tiveram redução proporcional também até a metade do período, passando de 27,2 a 22,5\%, depois crescendo até 33,6\% em 2013. Já as internações por transtornos psicóticos não orgânicos (F2) demonstraram redução linear estatisticamente significativa ao longo do período, decrescendo de 47,6\%, em 2001, para 40,6\%, em 2013. Os transtornos do humor (F3) mostraram tendência cúbica, com elevação relativa acentuada nos primeiros quatro anos (de 10,7\% para $16,6 \%)$, redução até $2011(13,4 \%)$ e suave elevação até 2013 (15,5\%). Para os transtornos neuróticos $(\mathrm{F} 4)$, houve relativa elevação nos primeiros cinco anos, passando de 0,9 a 2,3\%, seguida de redução até $0,5 \%$, em 2013 . Para todas essas tendências descritas, os modelos de regressão revelaram coeficientes de determinação entre 61 e $80 \%$ e $p<0,003$. As proporções de internações por transtornos do desenvolvimento (F7), bem como as do grupo de outros diagnósticos não citados, não demonstraram tendência à alteração ao longo do período.

Enquanto nos hospitais públicos ocorreu inversão da nosologia prevalente, cujas interna- ções por transtornos ligados ao uso de substâncias superaram (a partir de 2011) aquelas por transtornos psicóticos não orgânicos, o mesmo não se deu no subgrupo dos hospitais privados conveniados ao SUS. Nestes últimos, os transtornos psicóticos se mantiveram como os mais prevalentes ao longo de todo o período do estudo (Figura 3).

\section{Discussão}

Diante das recentes mudanças provocadas pelo redirecionamento da atenção à saúde mental no Brasil, faz-se necessário conhecer a amplitude e o alcance das alterações ocorridas. Este trabalho propôs a análise de um grande banco de dados que possibilitou o conhecimento do perfil das internações ocorridas nos hospitais psiquiátricos especializados com leitos credenciados pelo Sistema Único de Saúde do estado de Minas Gerais, ao longo de 13 anos, a partir da aprovação da Lei no $10.216 / 2001^{14}$.

De nosso conhecimento, este é o primeiro estudo a analisar a evolução das internações pelo SUS ocorridas em todos os hospitais psiquiátricos mineiros, no âmbito da reforma da assistência à saúde mental.

A análise das séries temporais evidenciou efetiva modificação nas características das internações psiquiátricas pelo SUS, no período do estudo, no estado de Minas Gerais. O perfil nosológico apresentou mudanças significativas, notadamente a elevação das proporções das internações

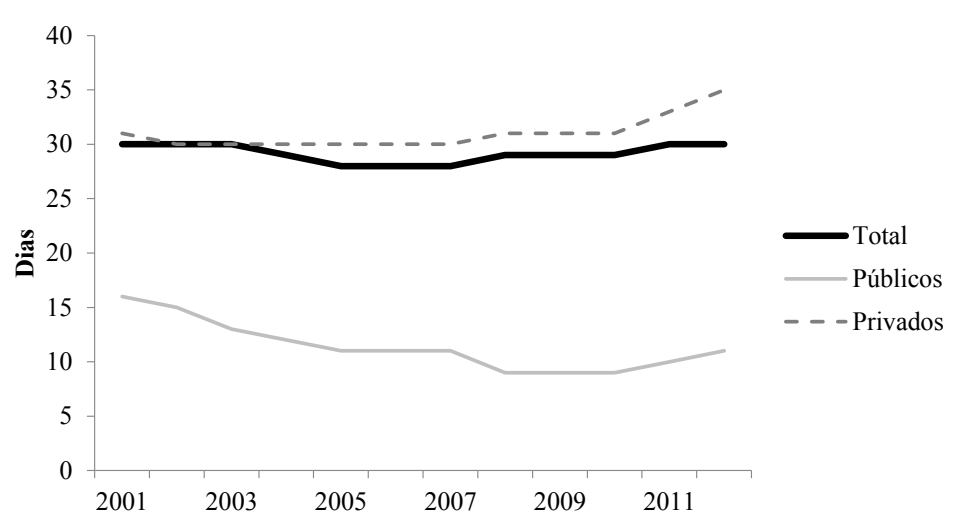

Figura 2. Evolução das medianas do tempo de permanência em hospitais psiquiátricos de Minas Gerais, 20012012, segundo a natureza jurídica dos hospitais. 

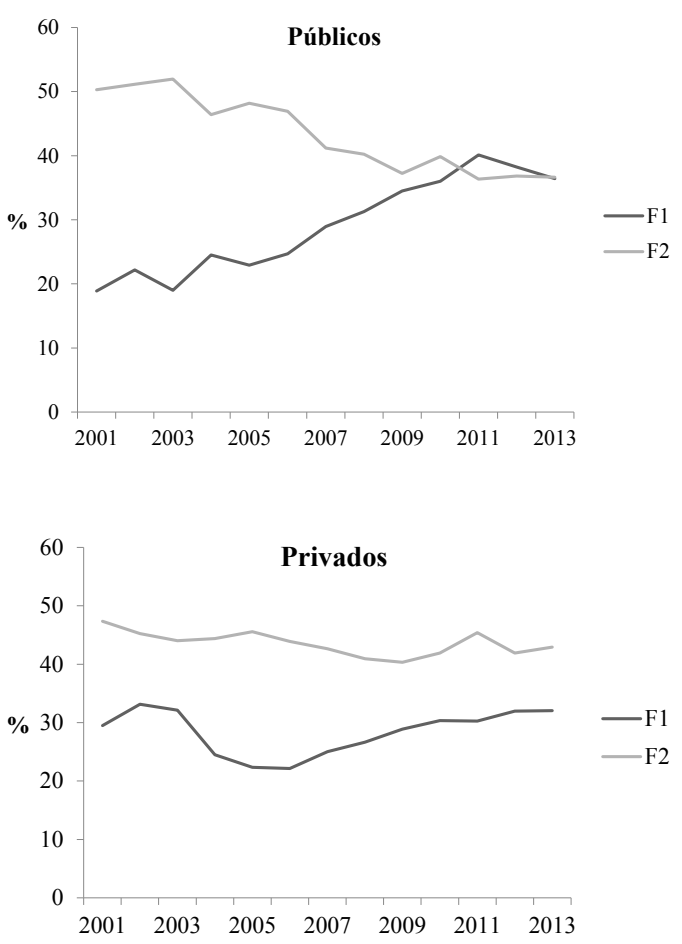

Figura 3. Evolução das nosologias prevalentes nos hospitais públicos e privados de Minas Gerais, 20012013.

( $\mathrm{F} 1=$ tr. mentais e de comportamento decorrentes do abuso/ dependência de substâncias psicoativas; F2=esquizofrenia, tr. esquizotípicos e delirantes)

por transtornos ligados ao abuso de substâncias psicoativas e de transtornos do humor como diagnóstico principal. Essa variação se acompanhou da redução das proporções de internações por transtornos psicóticos e de transtornos neuróticos. Mudanças no perfil nosológico foram relatadas em outros estudos nacionais no cenário da reforma. Quando apenas os hospitais psiquiátricos públicos de Belo Horizonte-MG foram analisados $^{4}$, observou-se relativa redução das internações por transtornos psicóticos, acompanhadas de elevação das proporções das internações por transtornos do humor e por abuso de substâncias, entre 2002 e 2011. As tendências observadas na capital são semelhantes às do estado de Minas como um todo, embora apresentem magnitudes diferentes.

Usando metodologia semelhante à do presente estudo, Candiago e Abreu ${ }^{5}$ também obtiveram relativo aumento nas internações por transtornos do humor e redução daquelas por trans- tornos psicóticos, no período de 2000 a 2004, nas internações pelo SUS no estado do Rio Grande do Sul. Já outro estudo ${ }^{6}$, conduzido em enfermarias psiquiátricas de hospital geral universitário e um único hospital psiquiátrico de Ribeirão Preto-SP, reportou elevação na proporção de internações por transtornos depressivos, transtornos da personalidade e ligados a abuso de drogas, no período de 2000 a 2007. O perfil de pacientes admitidos em leitos psiquiátricos de hospital geral é diverso daquele dos hospitais psiquiátricos ${ }^{15}$, o que pode explicar a discrepância nas tendências verificadas naquela amostra em relação às do presente estudo.

Duas fases podem ser identificadas nessas tendências temporais relativas ao perfil nosológico: antes e após 2005. Na primeira fase, houve redução das proporções das internações por F1 e F2 e aumento das por F0, F3 e F4. Na segunda fase, aumentam-se as proporções por F1 e se reduzem as por F0, F2 e F4. Com os dados disponíveis, é difícil tecer hipóteses sobre as razões para esse fenômeno. O período coincide com a implementação do Programa Nacional de Avaliação do Sistema Hospitalar/Psiquiatria ${ }^{16}$ e do Programa Anual de Reestruturação da Assistência Hospitalar no SUS ${ }^{17,18}$, que resultaram no descredenciamento dos macro-hospitais e das unidades de atenção a pacientes de longa permanência, onde é mais provável que permaneçam os pacientes portadores de psicoses crônicas do que os dependentes químicos.

De fato, existiu diferença na evolução temporal das proporções das internações por F1 e F2 entre os hospitais públicos e os privados. Enquanto nas instituições públicas a proporção de internações por F1 cresceu consistentemente desde 2001, nos hospitais privados houve relativo decréscimo até 2006, seguido de elevação até 2013. A inversão da nosologia prevalente, em que os transtornos ligados ao abuso e dependência de substâncias psicoativas superaram os transtornos psicóticos, só se observou nos hospitais públicos. É possível que essa diferença se associe a fatores ligados à rede de assistência e às práticas hospitalares propriamente ditas. Em Belo Horizonte, onde se concentra o maior volume de internações em hospitais públicos, existe uma rede abrangente de CAPS e de unidades básicas de saúde. Pode-se hipotetizar que essa rede extra -hospitalar na capital mineira tenha sido capaz de reter os pacientes psicóticos em tratamento externo, mais do que os dependentes químicos. Coelho et al. ${ }^{4}$ pressupõem que o aumento relativo nas internações por dependências químicas, 
em relação às psicoses, na capital do estado, estivesse relacionado não apenas à diferença notável na efetividade dos tratamentos direcionados a esses grupos diagnósticos, como também a uma relativa escassez de serviços extra-hospitalares especializados no tratamento dos transtornos ligados ao abuso de substâncias.

A idade média dos pacientes não se alterou significativamente ao longo do período de estudo, bem como se manteve a predominância do gênero masculino, apesar das mudanças ocorridas no perfil nosológico. Estes achados são corroborados pelos resultados de Silva ${ }^{19}$, Brenner et al. ${ }^{20} \mathrm{e}$ Strejilevich et al. ${ }^{21}$. É interessante notar que no mesmo período a população mineira envelheceu: houve significativas reduções da representação das faixas etárias de crianças e adolescentes e significativos aumentos das de habitantes a partir dos 40 anos de idade ${ }^{15}$. Essa alteração demográfica não se acompanha do envelhecimento da população psiquiátrica internada, nem de aumento dos casos de transtornos mentais orgânicos. Entre outros fatores, uma possível explicação para essa discrepância é que em um censo cada indivíduo é contado uma única vez, mas quando se estudam internações, pode haver múltiplas por indivíduo. Pacientes psicóticos e dependentes químicos, bem como os indivíduos mais jovens, são particularmente propensos a reinternações psiquiátrica ${ }^{22,23}$. Por esse motivo, poderiam estar sobrerrepresentados na amostra em comparação à população geral.

A apreciação adequada do tempo de permanência hospitalar só é possível a partir do agrupamento das AIHs repetidas para uma mesma internação, uma vez que nos hospitais psiquiátricos não é raro que as permanências superem os 45 dias e as AIHs subsequentes nem sempre mantenham numeração original, conforme preconiza o MS na Portaria no 111, de 2001. Esse procedimento de agrupamento foi executado por Szabzon $^{13}$, mas foi desconsiderado por outros autores $^{24,25}$. Na comparação com as pesquisas que usaram metodologia de agrupamento de AIHs, Szabzon $^{13}$ encontrou redução das internações mais prolongadas de 2000 a 2010, em residentes no município de São Paulo.

A redução observada nos tempos medianos de permanência nos hospitais públicos não foi verificada nos privados conveniados ao SUS. Novamente, a localização geográfica diferenciada entre esses grupos de hospitais pode ser um confundidor, na medida em que os leitos dos hospitais públicos se concentram na capital do estado, onde existe uma rede de atenção extra-hospita- lar mais abrangente. Isso favoreceria o processo da alta hospitalar, conferindo mais segurança à transição do paciente para os serviços externos. No entanto, não se pode descartar um efeito de diferenças nas prioridades da gestão, em que as unidades públicas apresentassem mais comprometimento com a eficiência hospitalar, buscando alto índice de renovação de leitos para otimizar a capacidade de assistência.

Este estudo baseou-se na utilização de dados fornecidos pelo Sistema de Informação Hospitalar do Sistema único de Saúde (SIH/ SUS). Essa fonte de dados secundária já respaldou algumas pesquisas semelhantes. Candiago e Abreu $^{5}$ analisaram as características das internações psiquiátricas no Rio Grande do Sul, utilizando um banco de dados oriundo das AIHs. Pepe ${ }^{26}$ utilizou dados do SIH/SUS para avaliar as internações e reinternações psiquiátricas no estado do Rio de Janeiro e Szabzon ${ }^{13}$ utilizou-se também de dados do DATASUS em estudo exploratório das internações psiquiátricas na cidade de São Paulo. A validade das informações registradas nas $\mathrm{AIH}$ já foi investigada ${ }^{27,28}$, muito embora não se apliquem especificamente às internações psiquiátricas nem às codificações da CID-10. Esses autores descreveram índices de concordância quanto aos diagnósticos das AIHs e dos prontuários, variando de 0,72 a 0,98 . É razoável supor que as outras variáveis estudadas sejam ainda mais confiáveis que o diagnóstico ${ }^{29}$, pois envolvem dados mais diretos. Ressalta-se que a remuneração dos hospitais pelo procedimento da internação em Psiquiatria não sofre a influência do diagnóstico, logo, é improvável que os registros de diagnóstico sejam artificialmente modificados.

De qualquer forma, por se embasar em dados secundários, existe a possibilidade de erros de registro não sistemáticos. Outra limitação relacionada à origem dos dados secundários é a ausência de identificadores individuais nos registros disponibilizados pelo DATASUS. Assim, não é possível verificar com adequada precisão as internações subsequentes para um mesmo indivíduo, impossibilitando as análises sobre reinternações.

Por outro lado, a utilização de bancos de dados oficiais de internação (SIH/SUS) permite o estudo de grandes amostras por longos períodos observacionais. A partir das técnicas de agrupamento de AIHs já descritas e validadas na literatura $^{5,13,30}$, torna-se possível individualizar as internações e extrair diversas variáveis de interesse epidemiológico.

Por meio da utilização de dados secundários do Sistema de Informações Hospitalares, foi 
possível conhecer o perfil das internações psiquiátricas nos hospitais especializados do estado de Minas Gerais em um período marcado por mudanças legislativas, institucionais e culturais. Dessa forma, foram percebidas mudanças tanto no volume quanto nas características das admissões. Notavelmente, houve redução de $57 \%$ no número de internações entre 2001 e 2013, observando-se elevação da proporção das internações por transtornos ligados ao abuso de substâncias e redução daquelas por transtornos psicóticos.

O monitoramento dos impactos das políticas de saúde mental sobre as práticas terapêuticas e as dinâmicas dos serviços de saúde é uma atividade necessária para municiar a gestão com as informações necessárias para a tomada de decisão e a priorização das ações. O esforço despendido nessa empreitada de pesquisa de forma alguma esgota o tema, sendo necessárias reavaliações em diferentes contextos temporais e regionais.

\section{Colaboradores}

APM Lara e FM Volpe participaram igualmente de todas as etapas de elaboração do artigo. 


\section{Referências}

1. Brasil. Ministério da Saúde (MS). Saúde Mental em Dados. Brasília: MS; 2012.

2. Brasil. Ministério da Saúde (MS). Reforma psiquiátrica e política de saúde mental no Brasil. Documento apresentado à Conferência Regional de Reforma dos Serviços de Saúde Mental: 15 anos depois de Caracas. OPAS. Brasília: MS; 2005.

3. Brasil. Ministério da Saúde (MS). Portaria no 3.088, de 23 de dezembro de 2011. Institui a Rede de Atenção Psicossocial para pessoas com sofrimento ou transtorno mental e com necessidades decorrentes do uso de crack, álcool e outras drogas, no âmbito do Sistema Único de Saúde. Diário Oficial da União 2011; 26 dez.

4. Coelho VAA, Volpe FM, Diniz SSL, Silva EEM, Cunha CF. Alteração do perfil de atendimento dos hospitais psiquiátricos públicos de Belo Horizonte, Brasil, no contexto da reforma da assistência à saúde mental. Cien Saude Colet 2014; 19(8):3605-3616.

5. Candiago RH, Abreu PB. Uso do Datasus para avaliação dos padrões das internações psiquiátricas. Rio Grande do Sul. Rev Saúde Pública 2007; 41(5):821-829.

6. Barros REM. Re-internações Psiquiátricas - influência de variáveis sócio demográficas, clínicas e de modalidade de tratamento [tese]. São Paulo: Universidade de São Paulo; 2012.

7. Organização Mundial da Saúde. The World Health Report 2001. Mental Health: New Understanding, New Hope. Geneva: OMS; 2001. [cited 2014 May 23]. Available from: http://www.who.int/whr/2001/en/ whr01_po.pdf

8. Saraceno B, Asioli F, Tognoni G. Manual de saúde mental: guia básico para atenção primária. São Paulo: Hucitec; 1997.

9. Brasil. Ministério da Saúde (MS). Departamento de Informática do Sistema Único de Saúde (DATASUS) [online]. Brasília; [s.d.]. [cited 2015 Jul 18]. Available from: http://www2.datasus.gov.br/DATASUS/index. php

10. Brasil. Ministério da Saúde (MS). Cadastro Nacional de Estabelecimentos de Saúde (CNES). 2015. [cited 2015 Aug 27]. Available from: http://cnes.datasus.gov.br

11. Brasil. Ministério da Saúde (MS). A experiência brasileira em sistemas de informação em saúde. Brasília: MS, Organização Pan-Americana da Saúde, Fiocruz; 2009.

12. Organização Mundial de Saúde (OMS). CID-10: classificação estatística internacional de doenças e problemas relacionados à saúde. 7a ed. São Paulo: EDUSP; 2004.

13. Szabzon F. Perfil das internações psiquiátricas em São Paulo: Um estudo exploratório [dissertação]. São Paulo: Universidade de São Paulo; 2013.

14. Brasil. Lei no 10.216, de 06 de abril de 2001. Dispõe sobre a proteção e os direitos das pessoas portadoras de transtornos mentais e redireciona o modelo assistencial em saúde mental. Diário Oficial da União 2002; 11 jan.

15. Katz G, Durst R, Shufman E, Bar-Hamburger R, Grunhaus L. A comparative study of psychiatric inpatients in a general hospital and a psychiatric hospital in Israel: Demographics, psychopathological aspects and drug abuse patterns. Isr Med Assoc J 2011; 13(6):329332.
16. Brasil. Ministério da Saúde (MS). Portaria no 251, de 31 de janeiro de 2002. Estabelece diretrizes e normas para a assistência hospitalar em psiquiatria, reclassifica os hospitais psiquiátricos, define e estrutura a porta de entrada para as internações psiquiátricas na rede SUS e dá outras providências. Diário Oficial da União 2002; 1 fev.

17. Brasil. Ministério da Saúde (MS). Portaria no 52, de 20 de janeiro de 2004. Institui o Programa Anual de restruturação da Assistência Psiquiátrica Hospitalar no SUS. Diário Oficial da União 2004; 20 jan.

18. Brasil. Ministério da Saúde (MS). Portaria no 53, de 20 de janeiro de 2004. Cria novos procedimentos no âmbito do Plano Anual de Reestruturação da Assistência Psiquiátrica Hospitalar no SUS - 2004 e dá outras providências. Diário Oficial da União 2004; 20 jan.

19. Silva EC. O Sistema de Informações Hospitalares do Sistema Único de Saúde: subsídios para o uso de dados secundários em psiquiatria e saúde mental [dissertação]. São Paulo: Universidade de São Paulo; 2004.

20. Brenner MK, Cardoso VM, Oliveira RLV, Fensterseifer GP, Barraz ACG, Barros LB, Cardozo V. Mudanças na clientela de internados em hospital psiquiátrico. J Bras Psiquiatr 1999; 48(9):415-420.

21. Strejilevich S, Chan M, Triskier F, Orgambide S. Operative of a psychiatric internation unit in a general hospital of Health Public System in Buenos Aires City. Vertex 2002; 13(48):85-92.

22. Lorine K, Goenjian H, Kim S, Steinberg AM, Schmidt $\mathrm{K}$, Goenjian AK. Risk factors associated with psychiatric readmission. J Nerv Ment Dis 2015; 203(6):425-430.

23. Ziber N, Hornik-Lurie T, Lerner Y. Predictors of early psychiatric rehospitalization: a national case register study. Isr J Psychiatry Relat Sci 2011; 48(1):49-53.

24. Mello R, Furegato ARF. Internações psiquiátricas no Rio de Janeiro de 1996 a 2005. Rev Enferm UERJ 2007; 15(2):176-182.

25. Kilsztajn S, Lopes ES, Lima LZ, Rocha PAF, Carmo MSN. Leitos hospitalares e reforma psiquiátrica no Brasil. Cad Saúde Pública 2008; 24(10):2354-2362.

26. Pepe VLE. Internações e reinternações psiquiátricas no Estado do Rio de Janeiro [tese]. São Paulo: Universidade de São Paulo; 2002.

27. Veras CMT, Martins MS. A confiabilidade dos dados nos formulários de autorização de internação hospitalar (AIH), Rio de Janeiro, Brasil. Cad Saúde Pública 1994; 10(3):339-355.

28. Mathias TAF, Soboll MLMS. Confiabilidade de diagnósticos nos formulários de autorização de internação hospitalar. Rev Saude Publica 1998; 32(6):526-532.

29. Lebrão M L, Mello-Jorge MHP, Laurenti R. Morbidade hospitalar por lesões e envenenamentos. Rev Saúde Pública 1997; 31(4):26-37.

30. Portela MC, Schramm JMA, Pepe VLE, Noronha MF, Cianeli MP. Algoritmo para a composição de dados de internação a partir do Sistema de Informações Hospitalares do Sistema Único de Saúde (SIH/SUS). Cad Saúde Pública 1997; 13(4):771-774.

Artigo apresentado em 13/06/2017

Aprovado em 19/10/2017

Versão final apresentada em 21/10/2017 